\title{
Comparison of Ranson, Glasgow, MOSS, SIRS, BISAP, APACHE-II, CTSI Scores, IL-6, CRP, and Procalcitonin in Predicting Severity, Organ Failure, Pancreatic Necrosis, and Mortality in Acute Pancreatitis
}

\author{
Ajay K. Khanna, ${ }^{1}$ Susanta Meher, ${ }^{1}$ Shashi Prakash, ${ }^{1}$ Satyendra Kumar Tiwary, ${ }^{1}$ \\ Usha Singh, ${ }^{2}$ Arvind Srivastava, ${ }^{3}$ and V. K. Dixit ${ }^{4}$ \\ ${ }^{1}$ Department of General Surgery, Institute of Medical Sciences, Banaras Hindu University, Varanasi, Ultra Pradesh 221005, India \\ ${ }^{2}$ Department of Pathology, Institute of Medical Sciences, Banaras Hindu University, Varanasi, Ultra Pradesh 221005, India \\ ${ }^{3}$ Department of Radiodiagnosis, Institute of Medical Sciences, Banaras Hindu University, Varanasi, Ultra Pradesh 221005, India \\ ${ }^{4}$ Department of Gastroenterology, Institute of Medical Sciences, Banaras Hindu University, Varanasi, Ultra Pradesh 221005, India
}

Correspondence should be addressed to Ajay K. Khanna; akhannabhu@gmail.com

Received 18 May 2013; Accepted 28 August 2013

Academic Editor: Attila Olah

Copyright (c) 2013 Ajay K. Khanna et al. This is an open access article distributed under the Creative Commons Attribution License, which permits unrestricted use, distribution, and reproduction in any medium, provided the original work is properly cited.

Background. Multifactorial scorings, radiological scores, and biochemical markers may help in early prediction of severity, pancreatic necrosis, and mortality in patients with acute pancreatitis (AP). Methods. BISAP, APACHE-II, MOSS, and SIRS scores were calculated using data within $24 \mathrm{hrs}$ of admission, whereas Ranson and Glasgow scores after $48 \mathrm{hrs}$ of admission; CTSI was calculated on day 4 whereas IL-6 and CRP values at end of study. Predictive accuracy of scoring systems, sensitivity, specificity, and positive and negative predictive values of various markers in prediction of severe acute pancreatitis, organ failure, pancreatic necrosis, admission to intensive care units and mortality were calculated. Results. Of 72 patients, 31 patients had organ failure and local complication classified as severe acute pancreatitis, 17 had pancreatic necrosis, and 9 died (12.5\%). Area under curves for Ranson, Glasgow, MOSS, SIRS, APACHE-II, BISAP, CTSI, IL-6, and CRP in predicting SAP were 0.85, 0.75, 0.73, 0.73, 0.88, 0.80, 0.90 , and 0.91 , respectively, for pancreatic necrosis $0.70,0.64,0.61,0.61,0.68,0.61,0.75,0.86$, and 0.90 , respectively, and for mortality $0.84,0.83,0.77,0.76,0.86,0.83,0.57,0.80$, and 0.75 , respectively. Conclusion. CRP and IL-6 have shown a promising result in early detection of severity and pancreatic necrosis whereas APACHE-II and Ranson score in predicting AP related mortality in this study.

\section{Introduction}

Acute pancreatitis (AP) is defined as an inflammatory process of the pancreas with possible peripancreatic tissue and multiorgan involvement inducing multiorgan dysfunction syndrome (MODS) with an increased mortality rate [1]. The incidence of acute pancreatitis per 100,000 population ranges from 5 to 80 cases per year, with the highest incidence rates being seen in Finland and the USA [2].

According to the Atlanta Classification, severe acute pancreatitis (SAP) is defined as an AP associated with local and/or systemic complications. Atlanta classification is a clinically based classification defining AP, severity, and complications. Development of organ dysfunction within $72 \mathrm{~h}$ of symptom onset is defined as an early severe acute pancreatitis (ESAP). Early severe acute pancreatitis is characterized by a short course, progressive MODS, early hypoxemia, increased incidence of necrosis, infection, and abdominal compartment syndrome (ACS) [3]. Multiorgan dysfunction syndrome, the extent of pancreatic necrosis, infection, and sepsis are the major determinants of mortality in AP [4]. Pancreatic necrosis is considered as a potential risk for infection, which represents the primary cause of late mortality. Occurrence of acute respiratory (ARF), cardiovascular (CVF), and renal failures (RF) can predict the fatal outcome in SAP [5]. A wide range of mortality $(20 \%-60 \%)$ has been reported in 
SAP. AP occurs when pancreatic enzymes are prematurely activated inside the pancreas leading to autodigestion of the gland and local inflammation [6]. These enzymes can also reach the bloodstream, stimulating the production of inflammatory cytokines and tumor necrosis factor- $\alpha$ (TNF$\alpha$ ) from leukocytes. The release of those substances triggers an inflammatory cascade, which leads to the SIRS [7]. Accurate diagnosis of SAP on admission to the hospital is of paramount importance and there is, therefore, agreement about the need for finding predictors of severe disease to identify patients who are at risk of morbidity and death.

Severe acute pancreatitis implies the presence of organ failure, local complications, or pancreatic necrosis and associated disruption of the pancreatic blood supply [8]. Several prognostic markers have been developed for severity stratification in acute pancreatitis. Multifactorial scoring systems incorporating clinical and biochemical criteria for severity assessment have been in use for some decades. These include the 11 criteria described by Ranson et al. in the 1970s [9], the Glasgow score (eight criteria), [10], MOSS score (12 criteria), BISAP score ( 5 criteria), and the acute physiology and chronic health evaluation (APACHE II) score (14 criteria) [11]. The sensitivity and specificity of these scoring systems for predicting severe acute pancreatitis range between $55 \%$ and $90 \%$, depending on the cut-off number and the timing of scoring [12]. Limitations of these scoring systems have been either the inability to obtain a complete score until at least 48 hours into the illness (Ranson and Glasgow scores) or the complexity of the scoring system itself (APACHE II). The APACHE-II score has not been developed specifically for acute pancreatitis but has been proven to be an early and reliable tool.

Regarding imaging dynamic contrast-enhanced CT (DCT), it is the imaging modality of choice for staging acute pancreatitis and for detecting complications [13]. DCT has been shown to detect pancreatic parenchymal necrosis with a diagnostic sensitivity of $87 \%$ and an overall detection rate of $90 \%$ [13]. The morphologic severity of acute pancreatitis can be determined using a CT severity index (CTSI) that was developed by Balthazar and coworkers and then simplified and extended to monitor organ failure by Silverman, Banks, and colleagues in 2004 [13]. Comparison of the original CTSI with mortality showed a good correlation between higher CTSI values and mortality and morbidity, and this holds true for the modified CTSI. Furthermore, the modified CTSI correlates well with the length of hospital stay and the development of organ failure [13].

Among single biochemical markers, C-reactive protein (CRP) remains the most useful. Despite its delayed increase, peaking not earlier than $72 \mathrm{~h}$ after the onset of symptoms, it is accurate and widely available. As a single prognostic marker, an elevated C-reactive protein (CRP) concentration of greater than $150 \mathrm{mg} / \mathrm{L}$ indicates that acute pancreatitis has a complicated course with a sensitivity of $85 \%$ in the first $72 \mathrm{~h}$ after the onset of symptoms. Although detection of elevated CRP levels is sensitive for severe acute pancreatitis it is not specific for the disease, and other causes of inflammation such as cholangitis and pneumonia need to be ruled out before severity assessment by measurement of CRP [14]. Among them the proinflammatory cytokine interleukin 6
(IL-6) seems to be the most promising parameter for use in clinical routine. It has been proven to be significantly increased in severe acute pancreatitis in comparison with mild disease already on the day of admission to hospital [15], with a peak concentration on day 3 after the clinical onset of the disease, and therefore to be helpful for early severity stratification. Sensitivities of $69-100 \%$ with specificities in the range $70-86 \%$ for the detection of severe acute pancreatitis are reported [15]. As the concentrations increase earlier than those of acute-phase proteins, several clinical studies have addressed the usefulness of the early prediction of severe acute pancreatitis, resulting in promising results for interleukin 6. Procalcitonin (PCT) is another marker that has been evaluated as a prognostic indicator for pancreatitis. Procalcitonin, the biologically inactive propeptide of calcitonin, is a more rapid acute-phase reactant with the ability to indicate a status of bacterial or fungal infection and sepsis. Several studies have indicated its diagnostic value for the differentiation between mild and severe acute pancreatitis within the first $24 \mathrm{~h}$ of disease presentation [16], showing a sensitivity of $89 \%$ and a specificity of $82 \%$, in a recent meta-analysis, but with a significant heterogeneity between individual studies [17].

\section{Material and Methods}

The aim of this prospective study was to study the various prognostic markers like Ranson, Glasgow, APACHE II, MOSS, SIRS, BISAP, CTSI, IL-6, CRP, and procalcitonin in cases of acute pancreatitis and to analyze the comparison between various prognostic markers in prediction of severe acute pancreatitis (SAP), organ failure (OF), pancreatic necrosis $(\mathrm{PNec})$, length of hospital stay (LOHS), requirement of ICU admission (ICUA), and mortality in acute pancreatitis.

After approval by the Institutional Review Board, this prospective study included 72 patients who were clinically suspected to have acute pancreatitis in a single surgical unit in Department of General Surgery, IMS, BHU, in collaboration with the Department of Gastroenterology, Department of Pathology, Department of Radiology, and Causality services from July 2010 to July 2012. Informed and written consent was obtained from all patients. The diagnosis of acute pancreatitis (AP) was based on the presence of two of the following three features: (1) abdominal pain characteristic of AP, (2) serum amylase and/or lipase $\geq 3$ times the upper limit of normal, and (3) characteristic finding of AP on abdominal CT Scan.

Demographic, radiographic, and laboratory data were collected from all these patients. In all these patients the following prognostic markers were used to know the severity of the disease (SAP), pancreatic necrosis $(\mathrm{PNec})$, requirement of ICU admission, length of hospital stay (LOHS), and mortality: (1) Ranson score, (2) Glasgow score, (3) MOSS score, (4) SIRS score, (5) APACHE II score, (6) BISAP score, (7) CTSI score, (8) IL-6, (9) CRP, and (10) procalcitonin. BISAP score, APACHE II score, and multiple organ system score (MOSS) were calculated using data from the first 
24 hours of admission and Ranson and Glasgow scores were calculated using data in first 24 hours and after 48 hours of admission. Presence of features of systemic inflammatory response syndrome (SIRS) was noted within the first 24 hours of admission. $2 \mathrm{~mL}$ of blood sample was collected on day 1 for IL-6. Serum was extracted after centrifugation in the Department of Pathology and stored at $-72^{\circ} \mathrm{C}$. Another $2 \mathrm{~mL}$ of blood sample was collected for procalcitonin card test. It is a semiquantitative method for rapid calculation of procalcitonin value using B.R.A.H.M.S. PCT-Q card. For this, serum was extracted from the blood sample after centrifugation. One drop of serum was put into the card and reading was taken after 30 minutes. Color of the test bar was matched with color given on the card. Value was noted according to the colour coding. Value ranging from $<0.5 \mathrm{ng} / \mathrm{mL}$ up to $>10 \mathrm{ng} / \mathrm{mL}$. $>0.5 \mathrm{ng} / \mathrm{mL}$ is taken as the cut-off value for detection of severity of acute pancreatitis according to the previous literature. This test was done in 42 cases only. On day 2 another $2 \mathrm{~mL}$ of blood sample was collected for C-reactive protein (CRP) and stored as serum at $-72^{\circ} \mathrm{C}$ along with the samples of IL- 6 to determine the value at the end of the study. CECT was performed in required cases on day 4 to look for pancreatic necrosis (PNec), local complications, and possible aetiology of AP. CTSI score was noted after CT scan.

Patients were classified as mild AP and severe AP, based on the presence of organ failure for more than $48 \mathrm{hrs}$ and local complications. Organ failure included shock (systolic blood pressure $<90 \mathrm{mmHg}$ ), pulmonary insufficiency (arterial $\mathrm{PO}_{2}<60 \mathrm{mmHg}$ at room air or the need for mechanical ventilation), or renal failure (serum creatinine level $>2 \mathrm{mg} / \mathrm{dL}$ after rehydration or hemodialysis). PNec was assessed by CECT; evidence of PNec on CT was defined as lack of enhancement of pancreatic parenchyma with contrast. At the end of the study, values of IL- 6 and CRP were calculated using ELISA kit.

2.1. Statistics. Normally distributed continuous variables were expressed as means. At the selected cut-off scores, each predictive system was evaluated for significant relationship to the severity, organ failure, pancreatic necrosis, need for ICU admission, and mortality by two-by-two contingency tables. The diagnostic cut-off value was expressed as its sensitivity, specificity, positive predictive value, negative predictive value, accuracy, and the area under the curve (AUC) under the receiver-operator characteristic (ROC) curve. The predictive accuracy of each scoring system and biochemical marker was measured by the area under the receiver-operating curve (AUC). All statistical analysis was made with SPSS software version 16.

\section{Observation and Results}

3.1. Patient's Characteristics. Mean age of presentation was 40.5 years (range $18-76$ ) with $51.4 \%$ males and $91.7 \%$ Hindu. The etiologies of AP included biliary (64\%), alcoholic $(13 \%)$, idiopathic $(9 \%)$, hypertriglyceridemia (2\%), postERCP (2\%), and trauma (2\%) (Table 1).
TABLE 1: Etiology of acute pancreatitis.

\begin{tabular}{lcc}
\hline Etiology & No. of cases & Percentage \\
\hline Biliary & 44 & 61.1 \\
Alcoholic & 13 & 18.0 \\
Idiopathic & 9 & 12.5 \\
Hypertriglyceridemia & 2 & 2.8 \\
Post-ERCP & 2 & 2.8 \\
Traumatic & 2 & 2.8 \\
\hline
\end{tabular}

ERCP: endoscopic retrograde cholangiopancreatography.

Thirty-one patients (43.1\%) were diagnosed as having SAP (organ failure with local complications), twenty five patients (34.7\%) developed persistent organ failure, and seventeen patients (23.6\%) had evidence of pancreatic necrosis on CECT. The average length of hospital stay was 10 days. Nine patients $(12.5 \%)$ needed ICU admission and nine patients (12.5\%) died during hospitalization. 54 patients (75\%) underwent CECT abdomen on day-4. IL-6 and CRP were done in 60 patients of whom $46.7 \%$ had IL- 6 value of $\geq 50 \mathrm{pg} / \mathrm{mL}$ and $41.7 \%$ of cases had CRP value of $\geq 150 \mathrm{mg} / \mathrm{L}$. Table 2 shows patient characteristics of the study cohort.

3.2. Comparison of Scoring Systems in Predicting SAP, Organ Failure, Pancreatic Necrosis, Length of Hospital Stay, ICU Admission, and Mortality. In prediction of SAP according to the AUC (with 95\% CI) CRP (0.91 (0.83-0.99)) and IL-6 (0.90 (0.81-0.99)) had the highest accuracy, followed by APACHE II (0.88 (0.79-0.97)) and Ranson (0.85 (0.76-0.92)). Also for prediction of pancreatic necrosis according to AUC (with 95\% CI) CRP (0.90 (0.82-0.97)) and IL-6 (0.86 (0.77-0.94)) had the highest accuracy as compared to other markers, followed by CTSI $(0.75(0.59-0.91))$ and for prediction of mortality according to AUC (with 95\% CI) accuracy was highest for APACHE II 0.86 (0.77-0.95) followed by Ranson score $(0.84(0.75-0.94))$. AUCs for each scoring system in predicting SAP, PNEC, and mortality are shown in Table 3. Among the various markers CRP and IL- 6 had the highest accuracy in predicting both SAP and PNEC but for mortality APACHE II and Ranson showed a little higher accuracy than the above two markers.

Among the multifactorial scoring systems, APACHE II and Ransons score had highest accuracy for predicting SAP and PNEC (Figures 1(a) and 1(c)). CTSI score as expected had the highest accuracy for prediction of pancreatic necrosis among the scoring systems (Figure 1(b)).

On the basis of the highest sensitivity and specificity values generated from the receiver-operating characteristic curves, the following cut-offs were selected for further analysis. Ranson $\geq 3$, Glasgow $\geq 3$, MOSS $\geq 5$, BISAP $\geq 2$, APACHE II $\geq 8$, CTSI $\geq 5$, procalcitonin $\geq 0.5 \mathrm{ng} / \mathrm{mL}, \mathrm{CRP} \geq$ $150 \mathrm{mg} / \mathrm{L}$, and IL- $6 \geq 50 \mathrm{pg} / \mathrm{mL}$. The observed incidence of severe disease, organ failure, pancreatic necrosis, need for ICU admission, average length of hospital stay, and mortality stratified by the various markers with their cut-offs is given in the Table 4 . The number of patients with Ranson score $\geq 3$ was 35 , Glasgow $\geq 3$ was 31 , MOSS $\geq 5$ was 42 , APACHE II $\geq 8$ was 
TABLE 2: Patients characteristics.

\begin{tabular}{|c|c|c|}
\hline Patients characteristics & No. of cases & Percentage \\
\hline \multicolumn{3}{|l|}{ Sex } \\
\hline Male & 37 & 51.4 \\
\hline Female & 35 & 48.6 \\
\hline \multicolumn{3}{|l|}{ Religion } \\
\hline Hindu & 66 & 91.7 \\
\hline Muslim & 6 & 8.3 \\
\hline \multicolumn{3}{|l|}{ Age group } \\
\hline $11-20$ & 9 & 12.5 \\
\hline $21-30$ & 17 & 23.6 \\
\hline $31-40$ & 12 & 16.7 \\
\hline $41-50$ & 16 & 22.2 \\
\hline $51-60$ & 5 & 6.9 \\
\hline $61-70$ & 13 & 18.1 \\
\hline \multicolumn{3}{|l|}{ Comorbidities } \\
\hline Diabetes mellitus & 3 & 4.2 \\
\hline Hypertension & 1 & 1.4 \\
\hline Other & 2 & 2.8 \\
\hline No comorbid condition & 62 & 86.1 \\
\hline Both DM and hypertension & 4 & 5.6 \\
\hline \multicolumn{3}{|l|}{ BMI } \\
\hline$<18.5$ & 1 & 1.4 \\
\hline $18.5-24.9$ & 55 & 76.4 \\
\hline $25-29.9$ & 14 & 19.4 \\
\hline $30-34.9$ & 2 & 2.8 \\
\hline \multicolumn{3}{|l|}{ Presentations } \\
\hline Pain abdomen & 72 & 100 \\
\hline Radiating & 62 & 86.1 \\
\hline Nonradiating & 10 & 13.9 \\
\hline Peritonitis & 62 & 86.1 \\
\hline Localized & 36 & 58.1 \\
\hline Diffuse & 26 & 41.9 \\
\hline Nausea & 6 & 8.3 \\
\hline Vomiting & 51 & 70.8 \\
\hline Distension abdomen & 29 & 40.2 \\
\hline Nonpassage of flatus and stool & 21 & 29.2 \\
\hline Breathlessness & 22 & 30.6 \\
\hline
\end{tabular}

32 , SIRS was 39 , BISAP $\geq 2$ was 36 , procalcitonin $\geq 0.5 \mathrm{ng} / \mathrm{mL}$ was $24, \mathrm{CRP} \geq 150 \mathrm{mg} / \mathrm{L}$ was 25 , and IL- $6 \geq 50 \mathrm{pg} / \mathrm{mL}$ was 28.

IL-6, CRP, and procalcitonin have the highest sensitivity for prediction of SAP. The specificity, PPV, NPV, and accuracy of IL-6 and CRP are also very high for prediction of SAP. Regarding OF the sensitivity for prediction is very high for procalcitonin, IL-6, APACHE II, and Ransons score and CRP is more specific and more accurate in prediction of OF. CRP is highly sensitive and specific for prediction of $\mathrm{PNec}$ with a very high accuracy. IL-6 and CTSI scores are the next markers which had a very high sensitivity for prediction of PNec. There was a higher need of ICU admission in patients with SIRS and a high MOSS, APACHE and Ranson scores. Regarding mortality, multifactorial scoring systems
TABLE 3: AUC (area under curve) of different prognostic markers in predicting SAP, PNEC, and mortality.

\begin{tabular}{lccc}
\hline AUC (95\% CI) & SAP & PNEC & Mortality \\
\hline Ranson & $0.85(0.76-0.92)$ & $0.70(0.55-0.89)$ & $0.84(0.75-0.94)$ \\
Glasgow & $0.75(0.63-0.86)$ & $0.64(0.49-0.79)$ & $0.83(0.73-0.93)$ \\
MOSS & $0.73(0.61-0.85)$ & $0.61(0.46-0.77)$ & $0.77(0.62-0.92)$ \\
SIRS & $0.73(0.61-0.85)$ & $0.61(0.46-0.76)$ & $0.76(0.64-0.88)$ \\
APACHE II & $0.88(0.79-0.97)$ & $0.68(0.58-0.83)$ & $0.86(0.77-0.95)$ \\
BISAP & $0.80(0.71-0.91)$ & $0.61(0.47-0.72)$ & $0.83(0.69-0.97)$ \\
IL-6 & $0.90(0.81-0.99)$ & $0.86(0.77-0.94)$ & $0.80(0.69-0.91)$ \\
CRP & $0.91(0.83-0.99)$ & $0.90(0.82-0.97)$ & $0.75(0.63-0.88)$ \\
CTSI & $0.66(0.53-0.79)$ & $0.75(0.59-0.91)$ & $0.57(0.35-0.78)$ \\
\hline
\end{tabular}

MOSS: multiple organ system score, APACHE II: acute physiology and chronic health evaluation II, SIRS: systemic inflammatory response syndrome, BISAP: bedside index for severe acute pancreatitis, IL-6: interleukin 6, CRP: C-reactive protein, and CTSI: CT severity index.

(APACHE II, Ranson, SIRS, and Glasgow), procalcitonin, and IL-6 were more accurate in predicting mortality. IL-6 and APACHE II were more accurate in predicting mortality (Table 5).

\section{Discussion}

In this study we have compared all the scoring systems, biochemical and radiological markers for prediction of morbidity and mortality in acute pancreatitis. We confirmed that single biochemical markers can be used as a reliable indicator for early stratification of severity of acute pancreatitis within 24 hours of admission.

The overall mortality in our cohort was $12.5 \%$ and $43.1 \%$ of patients had SAP. As expected the proportion of patients with severe disease and mortality in our cohort was higher as compared to previous studies [18]; this is probably because of a more number of referred cases admitted in our hospital.

Ranson's score is composed of 11 measures that are recorded as binary values on admission and at $48 \mathrm{hrs}$, and its primary aim was to evaluate the function of early operative intervention in patients with AP. A composite score of 3 or more is commonly used to classify a patient as having severe disease. Studies confirmed sensitivity from $40 \%$ to $90 \%$ [19]. Glasgow score proposed by Imrie for both alcohol and biliary acute pancreatitis seems to be more precise than that of Ranson, with a sensitivity for the assessment of severe acute pancreatitis of 56\%-85\% [20] using 8 laboratory factors within the first $48 \mathrm{~h}$ of treatment to calculate it [10], and more than three positive criteria indicate severe acute pancreatitis. Though fewer markers are taken into account, this score as well as the Ranson score predicts severe acute pancreatitis. Another commonly used severity index is the APACHE II index $[21,22]$. This clinical tool measures the physiological response to injury and inflammation-driven stress and was initially designed to predict prolonged intensive care unit treatment and mortality. Papachristou et al. [18] found sensitivity, specificity, and accuracy of $84.2 \%, 89.8 \%$, and $94 \%$ of Ranson criteria for prediction of SAP and $70.3 \%$, $71.9 \%$ and $78 \%$ for APACHE II score. In our study we have 

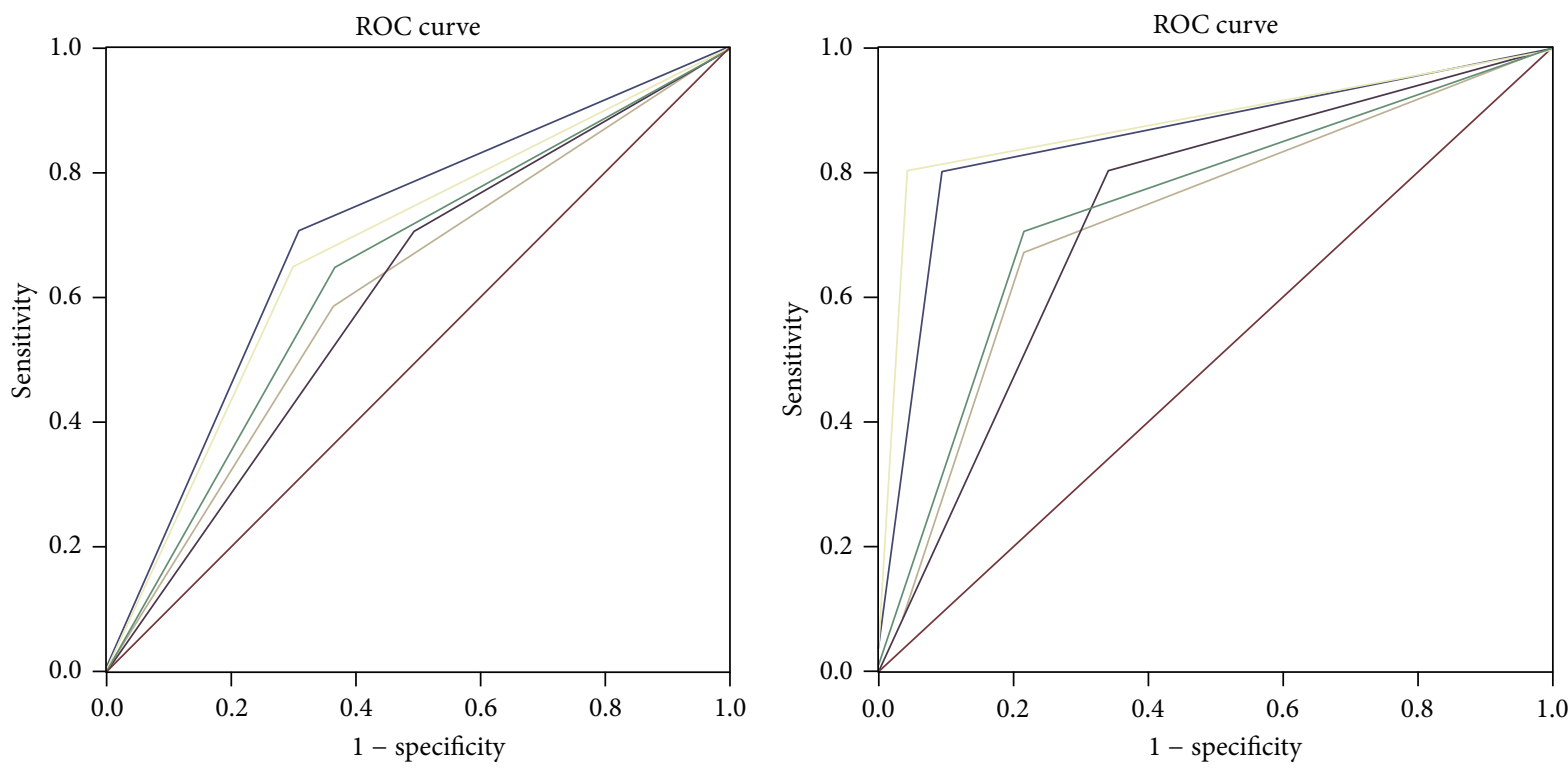

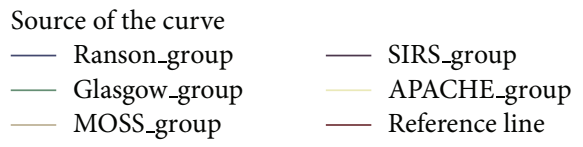

(a)

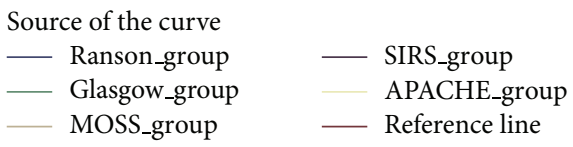

(b)

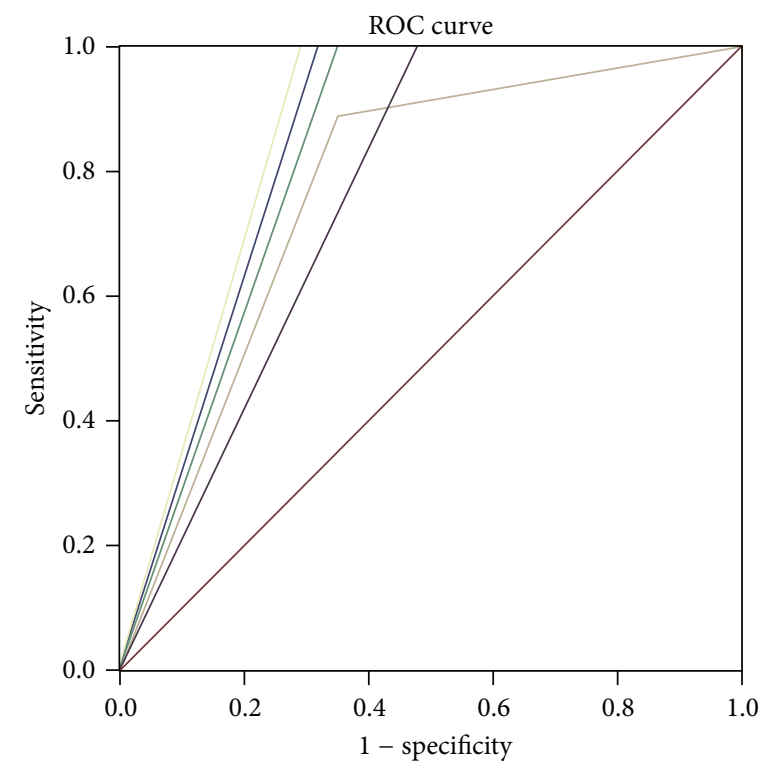

Source of the curve

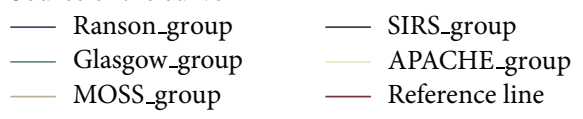

(c)

FIGURE 1: AUC comparison of various scoring systems in predicting SAP (a), pancreatic necrosis (b), and mortality (c). Diagonal segments are produced by ties.

found sensitivity, specificity, and accuracy of $83.9 \%, 78 \%$, and $85 \%$ of Ranson criteria for prediction of SAP and $80.6 \%$, $82.9 \%$, and $88 \%$ for APACHE II score. Similar result has been found for prediction of pancreatic necrosis and mortality. For Glasgow score we found 71\%, 78\%, and 75\% sensitivity, specificity and accuracy for prediction of SAP, respectively, which is in accordance with the study done by Blamey et al. [10].

BISAP and MOSS scores are newly developed prognostic scoring systems containing data that are frequently evaluated 
TABLE 4: Incidence of SAP, OF, PNec, ICUA, and mortality stratified by Ranson, Glasgow, MOSS, APACHE II, SIRS, BISAP scores, Procalcitonin, IL-6, and CRP.

\begin{tabular}{|c|c|c|c|c|c|c|c|}
\hline Markers & No. (\%) & $\%$ SAP $(n)$ & $\% \mathrm{OF}(n)$ & $\% \mathrm{PNec}(n)$ & \%ICUA $(n)$ & \%MORT $(n)$ & LOHS (days) \\
\hline \multicolumn{8}{|l|}{ Ranson } \\
\hline$<3$ & $37(51.4)$ & $13.5(5)$ & $5.4(2)$ & $13.5(5)$ & $2.7(1)$ & $0(0)$ & 8.24 \\
\hline$\geq 3$ & $35(48.6)$ & $74.3(26)$ & $65.7(23)$ & 34.3 (12) & $22.9(8)$ & $25.7(9)$ & 12.08 \\
\hline Total & $72(100)$ & $43.1(31)$ & $34.7(25)$ & $23.6(17)$ & $12.5(9)$ & $12.5(9)$ & 10.1 \\
\hline \multicolumn{8}{|l|}{ Glasgow } \\
\hline$<3$ & $41(56.9)$ & $22.0(9)$ & $14.6(6)$ & $14.6(6)$ & $2.4(1)$ & $0(0)$ & 8.14 \\
\hline$\geq 3$ & $31(43.1)$ & $71.0(22)$ & $61.3(19)$ & $35.5(11)$ & $25.8(8)$ & $29.0(9)$ & 12.7 \\
\hline Total & $72(100)$ & $43.1(31)$ & $34.7(25)$ & $23.6(17)$ & $12.5(9)$ & $12.5(9)$ & 10.1 \\
\hline \multicolumn{8}{|l|}{ MOSS } \\
\hline$<5$ & $30(41.7)$ & $23.3(7)$ & $13.3(4)$ & $16.7(5)$ & $0(0)$ & $0(0)$ & 8.5 \\
\hline$\geq 5$ & $42(58.3)$ & $57.1(24)$ & $50.0(21)$ & $28.6(12)$ & $21.4(9)$ & $21.4(9)$ & 11.23 \\
\hline Total & $72(100)$ & $43.1(31)$ & $34.7(25)$ & $23.6(17)$ & $12.5(9)$ & $12.5(9)$ & 10.1 \\
\hline \multicolumn{8}{|l|}{ APACHE II } \\
\hline$<8$ & $40(55.6)$ & $15.0(6)$ & $2.5(1)$ & $15.0(6)$ & $2.5(1)$ & $0(0)$ & 8.22 \\
\hline$\geq 8$ & $32(44.4)$ & $78.1(25)$ & $75.0(24)$ & 34.4 (11) & $25.0(8)$ & $28.1(9)$ & 12.48 \\
\hline Total & $72(100)$ & $43.1(31)$ & $34.7(25)$ & $23.6(17)$ & $12.5(9)$ & $12.5(9)$ & 10.1 \\
\hline \multicolumn{8}{|l|}{ SIRS } \\
\hline Absent & $33(45.8)$ & $18.2(6)$ & $12.1(4)$ & $15.2(5)$ & $0(0)$ & $0(0)$ & 7.84 \\
\hline Present & $39(54.2)$ & $64.1(25)$ & $53.8(21)$ & $30.8(12)$ & $23.1(9)$ & $23.1(9)$ & 12.02 \\
\hline Total & $72(100)$ & $43.1(31)$ & $34.7(25)$ & $23.6(17)$ & $12.5(9)$ & $12.5(9)$ & 10.1 \\
\hline \multicolumn{8}{|l|}{ BISAP } \\
\hline$<2$ & $36(50)$ & $22.2(8)$ & $13.9(5)$ & $19.4(7)$ & $5.6(2)$ & $2.7(1)$ & 7.5 \\
\hline$\geq 2$ & $36(50)$ & $63.9(23)$ & $55.6(20)$ & $27.8(10)$ & $19.4(7)$ & $22.8(8)$ & 10.2 \\
\hline Total & $72(100)$ & $43.1(31)$ & $34.7(25)$ & $23.6(17)$ & $12.5(9)$ & $12.5(9)$ & 10.1 \\
\hline \multicolumn{8}{|l|}{ CTSI } \\
\hline$<5$ & $23(42.6)$ & $39.1(9)$ & $34.8(8)$ & $17.4(4)$ & $13.0(3)$ & $8.7(2)$ & 8.6 \\
\hline$\geq 5$ & $31(57.4)$ & $54.8(17)$ & $41.9(13)$ & $38.7(12)$ & $12.9(4)$ & $16.1(5)$ & 11.22 \\
\hline Total & $54(100)$ & $48.1(26)$ & $38.9(21)$ & $29.6(16)$ & $13.5(7)$ & $13.5(7)$ & 9.91 \\
\hline \multicolumn{8}{|l|}{ Procalcitonin } \\
\hline$<0.5 \mathrm{ng} / \mathrm{mL}$ & $18(42.9)$ & $16.7(3)$ & $0(0)$ & $16.7(3)$ & $5.6(1)$ & $0(0)$ & 9.19 \\
\hline$>0.5 \mathrm{ng} / \mathrm{mL}$ & $24(57.1)$ & 79.7 (19) & $70.8(17)$ & $45.8(11)$ & $20.8(5)$ & $29.2(7)$ & 11.5 \\
\hline Total & $42(100)$ & $52.4(22)$ & $40.5(17)$ & $33.3(14)$ & $14.3(6)$ & $16.7(7)$ & 10.92 \\
\hline \multicolumn{8}{|l|}{ IL-6 } \\
\hline$<50 \mathrm{pg} / \mathrm{mL}$ & $32(53.3)$ & $6.2(2)$ & $3.1(1)$ & $3.1(1)$ & $3.1(1)$ & $0(0)$ & 7.68 \\
\hline$\geq 50 \mathrm{pg} / \mathrm{mL}$ & $28(46.7)$ & $96.4(27)$ & $78.6(22)$ & $57.1(16)$ & $25.0(7)$ & $32.1(9)$ & 13.7 \\
\hline Total & $60(100)$ & $48.3(29)$ & $38.3(23)$ & $23.6(17)$ & $13.3(8)$ & $12.5(9)$ & 10.69 \\
\hline \multicolumn{8}{|l|}{ CRP } \\
\hline$<150 \mathrm{mg} / \mathrm{L}$ & $35(58.3)$ & $11.4(4)$ & $11.4(4)$ & $0(0)$ & $5.7(2)$ & $8.6(3)$ & 8.54 \\
\hline$\geq 150 \mathrm{mg} / \mathrm{L}$ & $25(41.7)$ & $100(25)$ & 76.0 (19) & $68.0(17)$ & $24.0(6)$ & $24.0(6)$ & 13.28 \\
\hline Total & $60(100)$ & $48.3(29)$ & $38.3(23)$ & $23.6(17)$ & $13.3(8)$ & $12.5(9)$ & 10.91 \\
\hline
\end{tabular}

SAP: severe acute pancreatitis, OF: organ failure, PNec: pancreatic necrosis, ICUA: intensive care unit admission, LOHS: length of hospital stay, MOSS: multiple organ system score, APACHE II: acute physiology and chronic health evaluation II, SIRS: systemic inflammatory response syndrome, BISAP: bedside index for severe acute pancreatitis, IL-6: interleukin 6, CRP: C-reactive protein, and CTSI: CT severity index.

at the time of admission which are accurate in predicting patient's outcome [23]. BISAP and MOSS scores have the advantage over Ranson and Glasgow scores of being calculated within $24 \mathrm{hrs}$ of admission. BISAP score is higher in patients having SIRS, in older patients and in patients with altered mental status, whereas Ranson score seems to perform accurate prediction of persistent organ failure (sensitivity 92\%, specificity 74.5\%, PPV 65.7\%, and NPV 94.6\%). BISAP has the disadvantage that it cannot easily distinguish transient from persistent organ failure. SIRS is one of the leading events responsible for the mortality of AP. In our study we have a sensitivity of $80.6 \%, 84 \%$, and $100 \%$ for prediction of SAP, OF, and mortality and an accuracy of $73 \%, 61 \%$, and $76 \%$, respectively. 
TABLE 5: Sensitivity, specificity, PPV, NPV, and accuracy of different markers in predicting SAP, OF, PNec, need for ICU admission, and mortality.

\begin{tabular}{|c|c|c|c|c|c|c|}
\hline & Sensitivity & Specificity & PPV & NPV & Accuracy & Kappa (95\% CI) \\
\hline \multicolumn{7}{|c|}{ Severe acute pancreatitis } \\
\hline Ranson & 83.9 & 78.0 & 74.3 & 86.5 & 80.6 & $0.61(0.40-0.75)$ \\
\hline Glasgow & 71.0 & 78.0 & 71.0 & 78.0 & 75.0 & $0.49(0.27-0.66)$ \\
\hline MOSS & 77.4 & 56.1 & 57.1 & 76.7 & 65.3 & $0.32(0.10-0.49)$ \\
\hline APACHE II & 80.6 & 82.9 & 78.2 & 85.0 & 81.9 & $0.63(0.42-0.78)$ \\
\hline SIRS & 80.6 & 65.9 & 64.1 & 81.8 & 72.2 & $0.45(0.23-0.61)$ \\
\hline BISAP & 74.2 & 68.3 & 63.4 & 77.8 & 70.8 & $0.42(0.19-0.59)$ \\
\hline CTSI & 65.4 & 50.0 & 54.8 & 60.9 & 57.4 & $0.15(-0.11-0.39)$ \\
\hline IL-6 & 93.1 & 96.8 & 96.4 & 93.8 & 95.0 & $0.90(0.73-1.0)$ \\
\hline CRP & 86.2 & 100 & 100 & 88.6 & 93.3 & $0.87(0.70-0.87)$ \\
\hline Procalcitonin & 86.4 & 75.0 & 79.2 & 83.3 & 81 & $0.62(0.33-0.79)$ \\
\hline \multicolumn{7}{|c|}{ Organ failure } \\
\hline Ranson & 92.0 & 74.5 & 65.7 & 94.6 & 80.6 & $0.61(0.42-0.69)$ \\
\hline Glasgow & 76.0 & 74.5 & 61.3 & 85.4 & 75.0 & $0.48(0.26-0.64)$ \\
\hline MOSS & 84.0 & 55.3 & 50.0 & 86.7 & 65.3 & $0.34(0.14-0.46)$ \\
\hline APACHE II & 96.0 & 60.9 & 49.0 & 97.5 & 70.8 & $0.44(0.30-0.48)$ \\
\hline SIRS & 84.0 & 61.7 & 53.8 & 87.9 & 69.4 & $0.40(0.20-0.53)$ \\
\hline BISAP & 80.0 & 66.0 & 55.6 & 86.1 & 70.8 & $0.42(0.20-0.56)$ \\
\hline CTSI & 65.2 & 45.5 & 45.5 & 65.2 & 53.6 & $0.10(-0.15-0.32)$ \\
\hline IL-6 & 95.7 & 33.3 & 78.6 & 75.0 & 78.1 & $0.35(0.03-0.50)$ \\
\hline CRP & 82.6 & 83.8 & 76.0 & 88.6 & 83.3 & $0.65(0.42-0.80)$ \\
\hline Procalcitonin & 100 & 72.0 & 70.8 & 100 & 83.3 & $0.68(0.45-0.68)$ \\
\hline \multicolumn{7}{|c|}{ Pancreatic necrosis } \\
\hline Ranson & 70.6 & 58.2 & 34.3 & 86.5 & 61.1 & $0.21(0.01-0.36)$ \\
\hline Glasgow & 64.7 & 63.6 & 35.5 & 85.4 & 63.9 & $0.22(0.01-0.39)$ \\
\hline MOSS & 70.6 & 45.5 & 28.6 & 83.3 & 51.4 & $0.11(-0.07-0.24)$ \\
\hline APACHE II & 64.7 & 61.8 & 34.4 & 85.0 & 62.5 & $0.20(-0.004-0.37)$ \\
\hline SIRS & 70.6 & 50.9 & 30.8 & 84.8 & 55.6 & $0.15(-0.04-0.29)$ \\
\hline BISAP & 58.8 & 52.7 & 27.8 & 80.6 & 54.2 & $0.08(-0.11-0.25)$ \\
\hline CTSI & 87.5 & 55.3 & 45.2 & 91.3 & 57.4 & $0.20(-0.31-0.36)$ \\
\hline IL-6 & 94.1 & 72.1 & 57.1 & 96.9 & 78.3 & $0.55(0.35-0.61)$ \\
\hline CRP & 100 & 81.4 & 68.0 & 100 & 86.7 & $0.71(0.53-0.71)$ \\
\hline Procalcitonin & 78.6 & 53.6 & 45.8 & 83.3 & 61.9 & $0.27(0.003-0.85)$ \\
\hline \multicolumn{7}{|c|}{ ICU admission } \\
\hline Ranson & 88.9 & 57.1 & 22.9 & 97.3 & 61.1 & $0.21(0.45-0.25)$ \\
\hline Glasgow & 88.9 & 63.5 & 25.8 & 97.6 & 66.7 & $0.26(0.09-0.31)$ \\
\hline MOSS & 100 & 47.6 & 21.4 & 100 & 54.2 & $0.19(0.06-0.19)$ \\
\hline APACHE II & 88.9 & 61.9 & 25.0 & 97.5 & 65.3 & $0.24(0.08-0.29)$ \\
\hline SIRS & 100 & 52.4 & 23.1 & 100 & 58.3 & $0.22(0.09-0.21)$ \\
\hline BISAP & 77.8 & 54.0 & 19.4 & 94.4 & 56.9 & $0.14(-0.01-0.20)$ \\
\hline CTSI & 87.5 & 55.3 & 45.2 & 91.3 & 64.8 & $0.001(-0.15-0.12)$ \\
\hline IL-6 & 87.5 & 59.6 & 25.0 & 96.9 & 63.3 & $0.23(0.05-0.29)$ \\
\hline CRP & 75.0 & 63.5 & 24.0 & 94.3 & 65.0 & $0.20(0.01-0.31)$ \\
\hline Procalcitonin & 83.3 & 47.2 & 20.8 & 94.4 & 52.4 & $0.14(-0.06-0.21)$ \\
\hline \multicolumn{7}{|c|}{ Mortality } \\
\hline Ranson & 100 & 58.7 & 25.7 & 100 & 63.9 & $0.26(0.12-0.26)$ \\
\hline Glasgow & 100 & 65.7 & 29.0 & 100 & 64.4 & $0.32(0.16-0.32)$ \\
\hline MOSS & 100 & 47.6 & 21.4 & 100 & 54.2 & $0.19(0.06-0.19)$ \\
\hline
\end{tabular}


TABLE 5: Continued.

\begin{tabular}{lcccccc}
\hline & Sensitivity & Specificity & PPV & NPV & Accuracy & Kappa (95\% CI) \\
\hline APACHE II & 100 & 63.5 & 28.1 & 100 & 68.1 & $0.30(0.15-0.30)$ \\
SIRS & 100 & 52.4 & 23.1 & 100 & 58.3 & $0.22(0.09-0.21)$ \\
BISAP & 88.9 & 55.6 & 22.2 & 97.2 & 59.7 & $0.19(0.04-0.24)$ \\
CTSI & 71.4 & 44.7 & 16.1 & 91.3 & 48.1 & $0.07(-0.10-0.16)$ \\
IL-6 & 100 & 62.7 & 32.1 & 100 & 68.3 & $0.34(0.16-0.34)$ \\
CRP & 66.7 & 62.7 & 24.0 & 91.4 & 63.3 & $0.17(-0.03-0.31)$ \\
Procalcitonin & 100 & 51.4 & 29.2 & 100 & 59.5 & $0.26(0.07-0.26)$ \\
\hline
\end{tabular}

SAP: severe acute pancreatitis, OF: organ failure, PNec: pancreatic necrosis, ICUA: intensive care unit admission, LOHS: length of hospital stay, MOSS: multiple organ system score, APACHE II: acute physiology and chronic health evaluation II, SIRS: systemic inflammatory response syndrome, BISAP: bedside index for severe acute pancreatitis, IL-6; interleukin 6, CRP: C-reactive protein, and CTSI: CT severity index.

Computed tomography severity index has shown a strong positive correlation with the development of complications and mortality in patients with AP $[12,24]$. It was developed by Balthazar et al. to evaluate the degree of pancreatic edema, necrosis and the presence of peripancreatic fluid collections. In the CTSI pilot study, a score of 7-10 was able to predict $92 \%$ morbidity and $17 \%$ mortality rate in patients with AP, compared to the low morbidity (2\%) and mortality (0\%) associated with a CTSI score of $0-1$ [25]. In our study we have found that CTSI had the highest sensitivity of $87.5 \%$ and $91.3 \%$ NPV among multifactorial scoring system in prediction of pancreatic necrosis as expected and the lowest sensitivity in prediction of organ failure (65.2\%).

C-Reactive protein (CRP) is an acute phase reactant produced by the liver in response to interleukin-1, interleukin6 , and tumor necrosis factor- $\alpha$ and it is the most widely available, low-cost, and well-studied marker of severity in AP. A cut-off level of $150 \mathrm{mg} / \mathrm{L}$ within the first $48 \mathrm{hrs}$ of symptom onset has sensitivity and specificity of $80-86 \%$ and $61-84 \%$, respectively, for SAP and accuracy $>80 \%$ for necrotizing pancreatitis [26], CRP was done in 60 patients in our study; $58.3 \%$ of patients were having CRP value $<150 \mathrm{mg} / \mathrm{L}$ whereas $41.7 \%$ of cases had value of $\geq 150 \mathrm{mg} / \mathrm{L}$. In patients having CRP level > $150 \mathrm{mg} / \mathrm{L}$ incidence of SAP, OF, PNEC, ICUA, MORT, and LOHS was found to be $100 \%$ (25), $76.0 \%$ (19), $68.0 \%(17), 24.0 \%$ (6), and $24.0 \%$ (6) with an average length of hospital stay of 13.8 days, respectively. In our study CRP had the highest sensitivity (100\%), NPV (100\%), and specificity $(81.4 \%)$ for pancreatic necrosis, followed by sensitivity of $86.2 \%$ and specificity and PPV of $100 \%$ for prediction of SAP. As a whole CRP is a good marker for prediction of complications and mortality in acute pancreatitis. The AUC for prediction for PNec was higher for CRP 0.90 (0.82-0.77).

Activated leukocytes release proinflammatory cytokines that stimulate the liver to produce acute phase proteins. Since the concentration of cytokines increases before acute phase proteins, numerous clinical studies have been done to assess the usefulness of cytokines, such as interleukin(IL-) 1, IL-6, IL-8, IL-10, and IL-18, in predicting severity early in the course of AP. Most trials have focused on the proinflammatory cytokines IL-6. Value of IL-6 is significantly elevated in SAP on the day of admission and tends to peak at $72 \mathrm{hrs}$ after the clinical onset of disease, which makes IL6 an excellent marker of early severity stratification. A 2009 meta-analysis, defining severity by the Atlanta Classification, revealed that the sensitivity and specificity ranges for IL-6 in the first three days of admission were $81-83.6 \%$ and $75.6-$ $85.3 \%$, respectively, with an IL- 6 AUC of 0.75 on day one and 0.88 on the second day of admission [24]. In our study IL-6 was done in 60 patients. $53.3 \%$ of patients were having IL-6 value $<50 \mathrm{pg} / \mathrm{mL}$ and $46.7 \%$ were having value of $\geq 50 \mathrm{pg} / \mathrm{mL}$. In patients having IL-6 level $>50 \mathrm{pg} / \mathrm{mL}$ incidence of SAP, OF, PNEC, ICUA, MORT, and average LOHS found was to be $96.4 \%$ (27), $78.6 \%$ (22), $57.1 \%$ (16), $25.0 \%$ (7), and $32.1 \%$ (9) with an average length of hospital stay of 13.7 days, respectively. IL- 6 has the highest sensitivity for prediction of SAP (93.1\%), organ failure (95.7\%), pancreatic necrosis (94.1\%), and mortality (100\%). Regarding specificity it has the highest specificity (96.8\%) for SAP. It has very high NPV (93.8\%) and accuracy (95.0\%) for prediction of SAP. It has very high NPV (100\%) for mortality and NPV (96.9\%) for prediction of pancreatic necrosis.

Procalcitonin (PCT) is a propeptide of the hormone calcitonin, which is released by hepatocytes, peripheral monocytes, and G-cells of the thyroid gland. PCT level can be measured by a semiquantitative strip test for fast results or by a fully automated assay to obtain a more accurate measurement. An increased PCT level has been found to be an early predictor of severity [27-29], pancreatic necrosis, and organ failure [30] in patients with AP. In a recent metaanalysis a subgroup of 8 studies using PCT cut-off values of $0.5 \mathrm{ng} / \mathrm{mL}$ as a discriminator found that the sensitivity and specificity of PCT for development of SAP were $73 \%$ and $87 \%$, respectively, with an overall AUC of 0.88 [31]. In our study procalcitonin has $100 \%$ sensitivity for prediction of organ failure and mortality with a sensitivity of $86.4 \%$ for prediction of SAP.

At the end of the study we found that for prediction of SAP IL-6 had the highest sensitivity of 93.1\%, followed by CRP $(86.2 \%)$, procalcitonin $(86.4 \%)$, and Ranson score (83.9\%). CRP had the highest specificity of $100 \%$ with an accuracy of $95 \%$, followed by IL-6 (96.4\%) with an accuracy of $95 \%$. Ranson and APACHE II scores come as the next best predictors of SAP. For prediction of organ failure procalcitonin had the highest sensitivity and NPV of $100 \%$, followed by IL-6, Ranson, and APACHE II scores. Accuracy for prediction of organ failure is the highest for procalcitonin $(83.3 \%)$ and APACHE II scores $(83.3 \%)$. For prediction of 
pancreatic necrosis it is the CRP which has the maximum sensitivity and NPV of $100 \%$, followed by IL- 6 which has a sensitivity of $94.1 \%$ and NPV of $96.9 \%$. Ranson and MOSS scores are the next best predictors of pancreatic necrosis. CTSI has a sensitivity of $87.5 \%$ for prediction of pancreatic necrosis. The AUC for pancreatic necrosis is the highest for CRP (0.90), followed by IL-6 (0.86), and Ranson score (0.70). Requirement of ICU admission was best predicted by MOSS score and SIRS with $100 \%$ sensitivity and NPV, followed by Ranson and Glasgow. Glasgow score has the highest accuracy for need of ICU requirement (66.7\%). For the mortality predictors it is IL-6, procalcitonin, Ranson, Glasgow, APACHE II, MOSS, and SIRS which have 100\% sensitivity and NPV; AUC for mortality prediction is the highest for Ranson (0.84) and APACHE II scores (0.86).

\section{Conclusion}

We conclude that determining the serum concentration of IL-6 on the first day and/or together with serum CRP concentration on the 2 nd day of admission is helpful in earlier prediction and assessment of the severity of acute pancreatitis taking into consideration the disadvantages of multifactorial scoring systems. However, there is no ideal single method in assessing the severity of the disease. Individual preference and available institutional facilities influence the method chosen for prognostic assessment of acute pancreatitis.

\section{Disclosure}

The authors disclose that this work bears no financial assistance from any source which requires to be mentioned.

\section{Conflict of Interests}

The authors do not have any conflict of interests to disclose.

\section{Authors' Contribution}

Professor Ajay K. Khanna, Professor Usha Singh, Professor Arvind Srivastava, and Professor V. K. Dixit carried out the study design. Dr. Susanta Meher and Dr. Shashi Prakash were responsible for data acquisitation. Professor Ajay K. Khanna, Dr. Susanta Meher, Dr. Shashi Prakash, Dr. Satyendra Kumar Tiwary, Professor Usha Singh, Professor Arvind Srivastava, and Professor V. K. Dixit analysed and interpreted the data. Dr. Susanta Meher and Dr. Shashi Prakash drafted the paper. Professor Ajay K. Khanna and Dr. Satyendra Kumar Tiwary revised the paper. Dr. Susanta Meher provided the statistical advice.

\section{References}

[1] I. A. Al Mofleh, "Severe acute pancreatitis: pathogenetic aspects and prognostic factors," World Journal of Gastroenterology, vol. 14, no. 5, pp. 675-684, 2008.
[2] P. A. Banks, "Epidemiology, natural history, and predictors of disease outcome in acute and chronic pancreatitis," Gastrointestinal Endoscopy, vol. 56, no. 6, pp. S226-S230, 2002.

[3] H.-Q. Tao, J.-X. Zhang, and S.-C. Zou, "Clinical characteristics and management of patients with early acute severe pancreatitis: Experience from a medical center in China," World Journal of Gastroenterology, vol. 10, no. 6, pp. 919-921, 2004.

[4] A. Buter, C. W. Imrie, C. R. Carter, S. Evans, and C. J. McKay, "Dynamic nature of early organ dysfunction determines outcome in acute pancreatitis," British Journal of Surgery, vol. 89, no. 3, pp. 298-302, 2002.

[5] L. Kong, N. Santiago, T.-Q. Han, and S.-D. Zhang, "Clinical characteristics and prognostic factors of severe acute pancreatitis," World Journal of Gastroenterology, vol. 10, no. 22, pp. 33363338, 2004.

[6] T. Hirano and T. Manabe, "A possible mechanism for gallstone pancreatitis: repeated short-term pancreaticobiliary duct obstruction with exocrine stimulation in rats," Proceedings of the Society for Experimental Biology and Medicine, vol. 202, no. 2, pp. 246-252, 1993.

[7] J. Norman, "Role of cytokines in the pathogenesis of acute pancreatitis," American Journal of Surgery, vol. 175, no. 1, pp. 7683, 1998.

[8] P. A. Banks, "Practice guidelines in acute pancreatitis," American Journal of Gastroenterology, vol. 92, no. 3, pp. 377-386, 1997.

[9] J. H. C. Ranson, K. M. Rifkind, D. F. Roses, S. D. Fink, K. Eng, and F. C. Spencer, "Prognostic signs and the role of operative management in acute pancreatitis," Surgery Gynecology and Obstetrics, vol. 139, no. 1, pp. 69-81, 1974.

[10] S. L. Blamey, C. W. Imrie, J. O’Neill, W. H. Gilmour, and D. C. Carter, "Prognostic factors in acute pancreatitis," Gut, vol. 25, no. 12, pp. 1340-1346, 1984.

[11] W. A. Knaus, E. A. Draper, D. P. Wagner, and J. E. Zimmerman, "APACHE II: a severity of disease classification system," Critical Care Medicine, vol. 13, no. 10, pp. 818-829, 1985.

[12] E. J. Balthazar, "Acute pancreatitis: assessment of severity with clinical and CT evaluation," Radiology, vol. 223, no. 3, pp. 603613, 2002.

[13] E. J. Balthazar, P. C. Freeny, and E. van Sonnenberg, "Imaging and intervention in acute pancreatitis," Radiology, vol. 193, no. 2, pp. 297-306, 1994.

[14] M. Büchler, P. Malfertheiner, C. Schoetensack, W. Uhl, W. Scherbaum, and H. G. Beger, "Value of biochemical and imaging procedures for the diagnosis of acute pancreatitis-results of a prospective clinical study," Zeitschrift fur Gastroenterologie, vol. 24, pp. 100-109, 1986.

[15] G. Sathyanarayan, P. K. Garg, H. K. Prasad, and R. K. Tandon, "Elevated level of interleukin-6 predicts organ failure and severe disease in patients with acute pancreatitis," Journal of Gastroenterology and Hepatology, vol. 22, no. 4, pp. 550-554, 2007.

[16] M.-L. Kylänpää-Bäck, A. Takala, E. A. Kemppainen et al., "Procalcitonin, soluble interleukin-2 receptor, and soluble Eselectin in predicting the severity of acute pancreatitis," Critical Care Medicine, vol. 29, no. 1, pp. 63-69, 2001.

[17] S. Purkayastha, A. Chow, T. Athanasiou et al., "Does serum procalcitonin have a role in evaluating the severity of acute pancreatitis? A question revisited," World Journal of Surgery, vol. 30, no. 9, pp. 1713-1721, 2006.

[18] G. I. Papachristou, V. Muddana, D. Yadav et al., "Comparison of BISAP, Ranson's, APACHE-II, and CTSI scores in predicting 
organ failure, complications, and mortality in acute pancreatitis," American Journal of Gastroenterology, vol. 105, no. 2, pp. 435-441, 2010.

[19] C. Wilson, A. Heads, A. Shenkin, and C. W. Imrie, "C-reactive protein, antiproteases and complement factors as objective markers of severity in acute pancreatitis," British Journal of Surgery, vol. 76, no. 2, pp. 177-181, 1989.

[20] N. J. M. London, J. P. Neoptolemos, J. Lavelle, I. Bailey, and D. James, "Contrast-enhanced abdominal computed tomography scanning and prediction of severity of acute pancreatitis: a prospective study," British Journal of Surgery, vol. 76, no. 3, pp. 268-272, 1989.

[21] Y. P. Yeung, B. Y. K. Lam, and A. W. C. Yip, "APACHE system is better than Ranson system in the prediction of severity of acute pancreatitis," Hepatobiliary and Pancreatic Diseases International, vol. 5, no. 2, pp. 294-299, 2006.

[22] M. Larvin and M. J. McMahon, "APACHE-II score for assessment and monitoring of acute pancreatitis," The Lancet, vol. 2, no. 8656, pp. 201-205, 1989.

[23] S. L. Taylor, D. L. Morgan, K. D. Denson, M. M. Lane, and L. R. Pennington, "A comparison of the ranson, glasgow, and APACHE II scoring systems to a multiple organ system score in predicting patient outcome in pancreatitis," American Journal of Surgery, vol. 189, no. 2, pp. 219-222, 2005.

[24] E. Aoun, J. Chen, D. Reighard, F. C. Gleeson, D. C. Whitcomb, and G. I. Papachristou, "Diagnostic accuracy of interleukin6 and interleukin- 8 in predicting severe acute pancreatitis: a meta-analysis," Pancreatology, vol. 9, no. 6, pp. 777-785, 2009.

[25] E. J. Balthazar, D. L. Robinson, A. J. Megibow, and J. H. C. Ranson, "Acute pancreatitis: value of CT in establishing prognosis," Radiology, vol. 174, no. 2, pp. 331-336, 1990.

[26] J. P. Neoptolemos, E. A. Kemppainen, J. M. Mayer et al., "Early prediction of severity in acute pancreatitis by urinary trypsinogen activation peptide: a multicentre study," The Lancet, vol. 355, no. 9219, pp. 1955-1960, 2000.

[27] I. S. Modrau, A. K. Floyd, and O. Thorlacius-Ussing, "The clinical value of procalcitonin in early assessment of acute pancreatitis," American Journal of Gastroenterology, vol. 100, no. 7, pp. 1593-1597, 2005.

[28] Y. Mandi, G. Farkas, T. Takacs, K. Boda, and J. Lonovics, "Diagnostic relevance of procalcitonin, IL-6, and sICAM-1 in the prediction of infected necrosis in acute pancreatitis," International Journal of Pancreatology, vol. 28, no. 1, pp. 41-49, 2000.

[29] N. Bülbüller, O. Doğru, R. Ayten, H. Akbulut, Y. S. Ilhan, and Z. Çetinkaya, "Procalcitonin is a predictive marker for severe acute pancreatitis," Ulusal Travma ve Acil Cerrahi Dergisi, vol. 12, no. 2, pp. 115-120, 2006.

[30] M.-L. Kylänpää-Bäck, A. Takala, E. Kemppainen, P. Puolakkainen, R. Haapiainen, and H. Repo, "Procalcitonin strip test in the early detection of severe acute pancreatitis," British Journal of Surgery, vol. 88, no. 2, pp. 222-227, 2001.

[31] R. Mofidi, S. A. Suttie, P. V. Patil, S. Ogston, and R. W. Parks, "The value of procalcitonin at predicting the severity of acute pancreatitis and development of infected pancreatic necrosis: systematic review," Surgery, vol. 146, no. 1, pp. 72-81, 2009. 


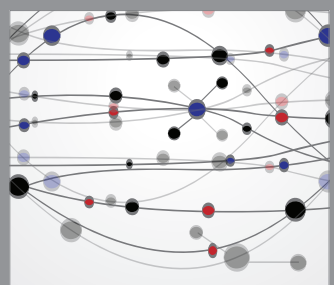

The Scientific World Journal
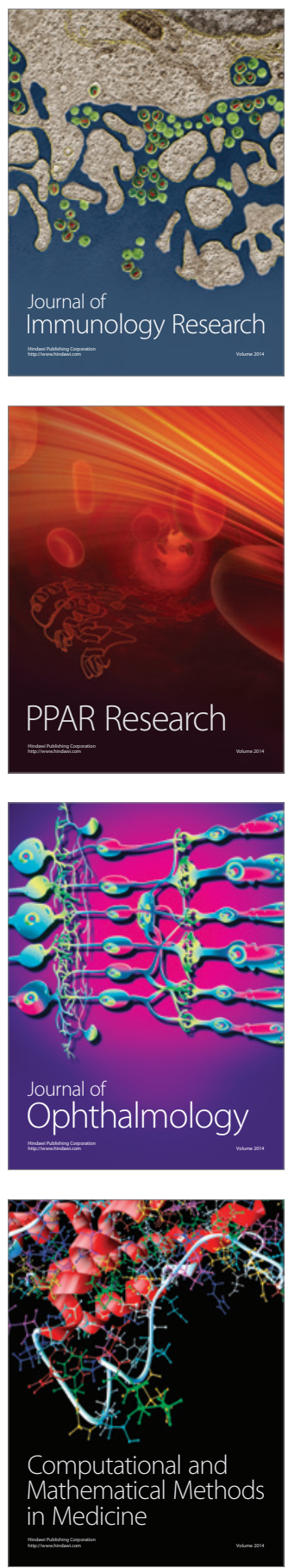

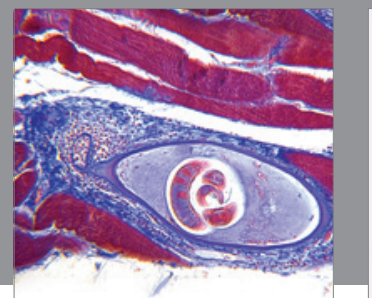

Gastroenterology

Research and Practice
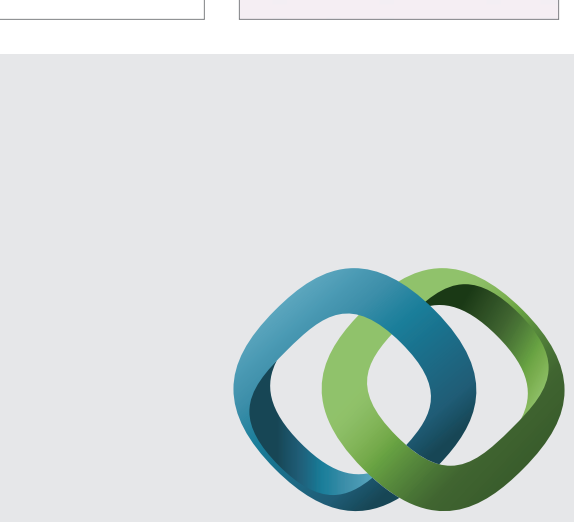

\section{Hindawi}

Submit your manuscripts at

http://www.hindawi.com
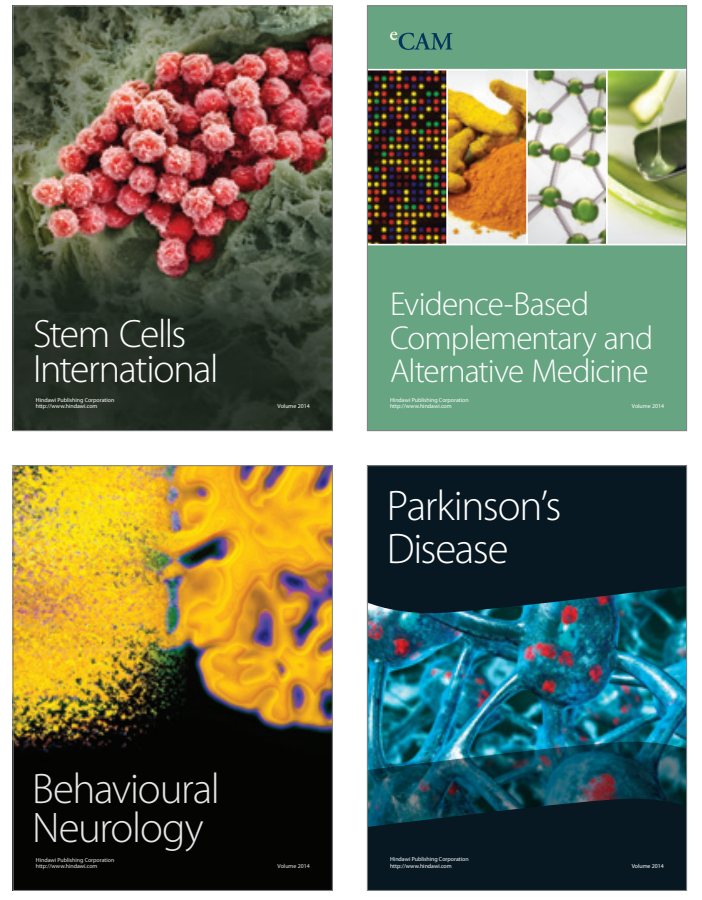
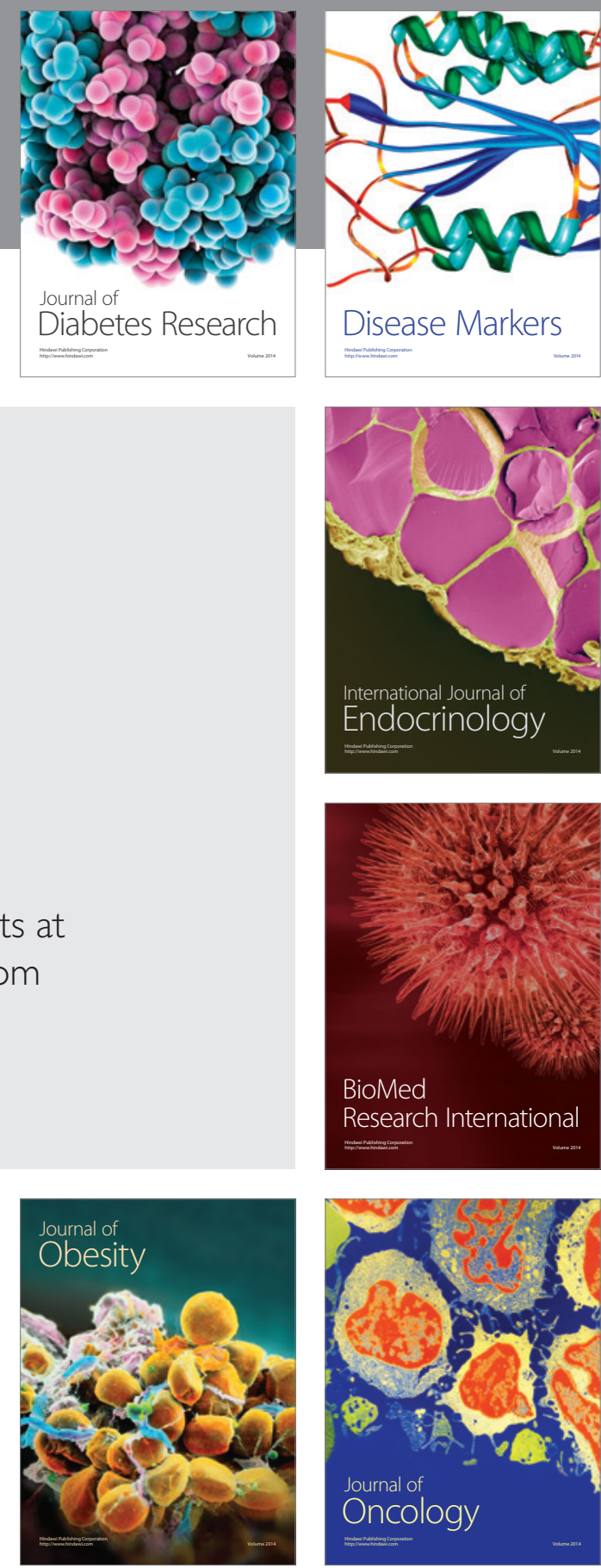

Disease Markers
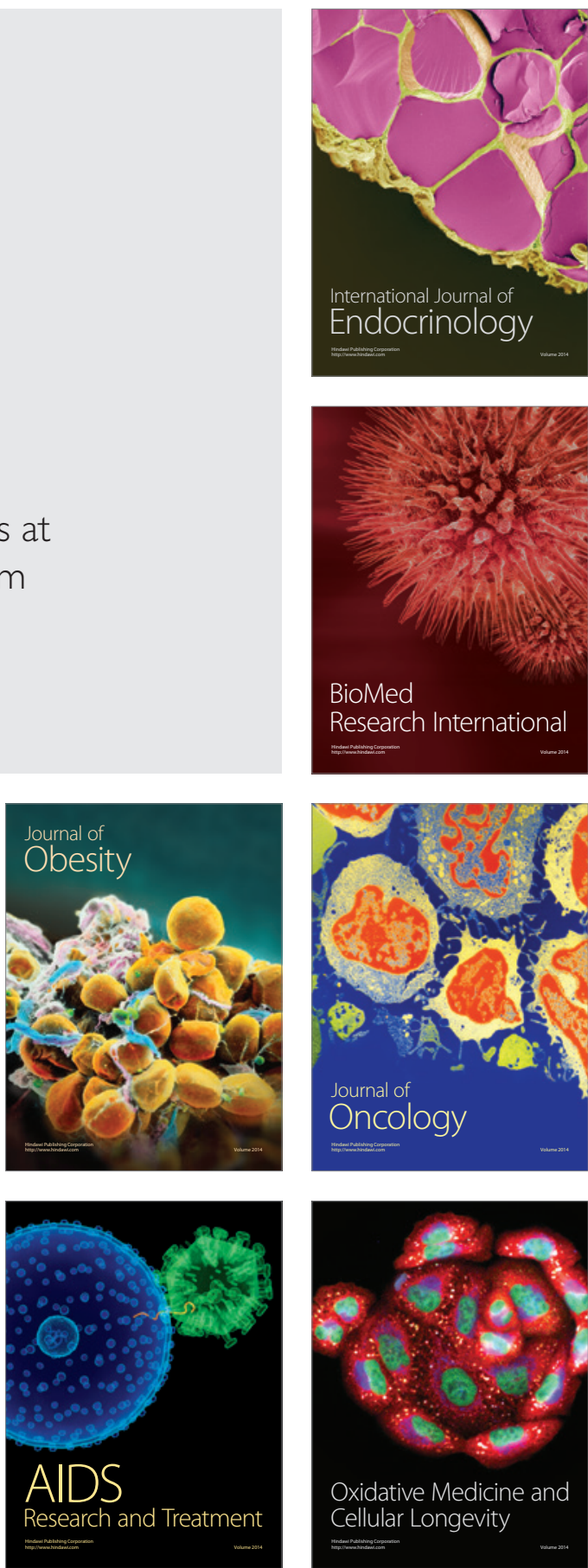